\title{
Kunst aus der «Seeseite» Münsterlingens
}

\section{Martina Wernli}

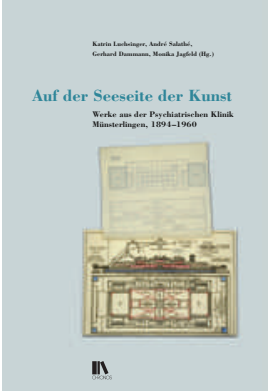

Katrin Luchsinger, André Salathé, Gerhard Dammann, Monika Jagfeld (Hg.) Auf der Seeseite der Kunst

Werke aus der Psychiatrischen Klinik Münsterlingen, 1894-1960 Zürich: Chronos-Verlag; 2015.

160 Seiten. $32 \mathrm{CHF}$. ISBN 978-3-0340-1262-1
Aus Anlass des 175-jährigen Jubiläums der Klinik in Münsterlingen, die ehemals «Irren- oder Heil- und Pflegeanstalt» hiess und die mittlerweile zur Spital Thurgau AG gehört, blickt die äusserst gelungene Begleitpublikation zu einer Ausstellung auf die Kunst zurück, die an diesem Ort entstanden ist. Mit der «Seeseite» bezeichnete die lokale Bevölkerung die psychiatrische Klinik, um sie von dem auf der Landseite gelegenen Kantonsspital zu unterscheiden und wohl auch, um mit einem euphemistischen Deckwort von einer Psychiatrie zu sprechen. Der Band widmet sich in einem Dutzend Beiträgen dem Fragenkomplex, welche Bedeutung künstlerisches Schaffen in einer Klinik haben kann. Aus dem grossen Zeitraum von 1894 bis 1960 finden sich (nur) rund 250 Werke in den Krankenakten. Die federführende Herausgeberin ist die Kunsthistorikerin Katrin Luchsinger, die das für die schweizerische Psychiatriegeschichte grundlegende und erfolgreiche Projekt «Bewahren besonderer Kulturgüter» leitete, mehrfach darüber publizierte

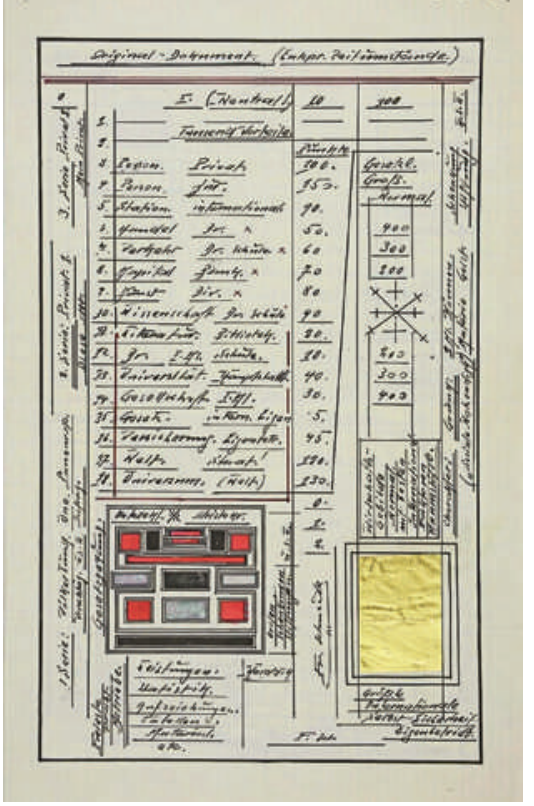

Franz Sch., "Grösste internationale SelbstSicherheit", 5.9.1948, $41,3 \times 36 \mathrm{~cm}$, Mischtechnik auf Papier, mit freundlicher Genehmigung des Staatsarchivs des Kantons Thurgau, Inv.-Nr. 156 recto.
Psychiater fokussiert (etwa Fallgeschichten und Kunstbetrachtungen Rorschachs oder Roland Kuhns, 19391980 Oberarzt und Direktor).

Im Band finden auch grundsätzliche Fragestellungen Platz, wie etwa die nach dem Umgang mit den Patienten- und Künstlernamen. Salathé verteidigt die Anonymisierung des Patientennamens aus einem Schutzgedanken gegenüber den damaligen Insassen heraus. Gegen diese Haltung wird im Band auch das Argument aufgeführt, dass es langsam Zeit sein könnte, diese Patienten beim Namen zu nennen, anstatt sie weiterhin im Status der «Infamen» festzuhalten. Im Weiteren geht es um das Verhältnis von Patientenakte und Kunstwerk - Salathé verteidigt hier die Integrität der Akte. Eine Ansicht, die sich in anderen Kliniken und Archiven leider nicht durchgesetzt hat, in denen Bilder gesondert von den Akten archiviert werden. Schliesslich geht es auch wieder um die seit Walter Morgenthaler und Hans Prinzhorn in den frühen 1920er Jahren diskutierte Frage, wie man diese und am meisten zur "Seeseite» beisteuerte. Hinzu kommen André Salathé, seines Zeichens Staatsarchivar des Kantons Thurgau, Gerhard Dammann, aktueller Klinikdirektor in Münsterlingen, und Monika Jagfeld, Direktorin des Museums im Lagerhaus in St.Gallen. Diese Gruppe und weitere Autoren/-innen - wie etwa der Historiker und Verantwortliche für das HermannRorschach-Archiv in Bern Urs Germann sowie dessen Vorgängerin Rita Signer oder die Kunsthistorikerin Kathrin Linder - stehen für unterschiedliche Perspektiven auf einen Forschungsgegenstand, der genauso facettenreich ist, wie es ihre Disziplinen sind. So werden neben den eigentlichen Kunstwerken beispielsweise auch fotografische Patientenaufnahmen Hermann Rorschachs (1909-1913 Assistenzarzt in Münsterlingen) analysiert, oder es wird auf das Schreiben einzelner
Werke benennen sollte - (Pathologische) Kunst, Art Brut, Bildnerei oder Werke von Klinikpatienten?

Zudem besticht der vom Chronos-Verlag gestaltete Band durch seine Bildreproduktionen, die den Lesern etwa die exakten geometrischen Darstellungen von Franz Sch. (eine trägt den wunderbaren Titel Grösste internationale Selbst-Sicherheit) näherbringen. Andere Zeichnungen, wie die von Karl H., kommen in der Reproduktion furchteinflössend zur Geltung. Die von der Kunstpädagogin Jacqueline Fahrni und Katrin Luchsinger zusammengestellten Kurzbiographien lassen hinter den anonymisierten Namen Teile von Lebensgeschichten und Schicksalen aufleuchten, die Werke werden dadurch situiert.

Ergänzend zu den historischen Werken zeigt das Kunstmuseum des Kantons Thurgau vom 13. September 2015 bis März 2016 aktuelle Arbeiten aus der Klinik. 